\title{
The Symmetry of Riemann $\xi$-Function
}

\author{
Chuanmiao Chen ${ }^{1,2}$ \\ ${ }^{1}$ School of Mathematics and Statistics, Central South University, Changsha, China \\ ${ }^{2}$ College of Mathematics and Statistics, Hunan Normal University, Changsha, China \\ Email:cmchen@hunnu.edu.cn
}

How to cite this paper: Chen, C.M. (2020) The Symmetry of Riemann $\xi$-Function. Advances in Pure Mathematics, 10, 464-470. https://doi.org/10.4236/apm.2020.108028

Received: June 28, 2020

Accepted: August 11, 2020

Published: August 14, 2020

Copyright $\odot 2020$ by author(s) and Scientific Research Publishing Inc. This work is licensed under the Creative Commons Attribution International License (CC BY 4.0).

http://creativecommons.org/licenses/by/4.0/

\section{Abstract}

To prove $\mathrm{RH}$, studying $\zeta$ and using pure analysis method likely are two kinds of the incorrect guide. Actually, a unique hope may study Riemann function $\xi(\tau)=u+i v, \tau=\beta+i t, \quad \beta=\sigma-1 / 2$ by geometric analysis, which has the symmetry: $v=0$ if $\beta=0$, and $v(t, \beta)=-\int_{0}^{\beta} u_{t}(t, r) \mathrm{d} r$. Assume that $|u|$ is single peak in each root-interval $I_{j}=\left[t_{j}, t_{j+1}\right]$ of $u$ for any fixed $\beta \in(0,1 / 2]$, using the slope $u_{t}$ of the single peak, we prove that $v$ has opposite signs at two end-points of $I_{j}$, there surely is an inner point so that $v=0$, so $\{|u|,|v| / \beta\}$ form a local peak-valley structure, and have positive lower bound $\|\xi\|=|u|+|v| / \beta \geq \mu\left(t_{j}, \beta\right)>0$ in $I_{j}$. Because each $t$ must lie in some $I_{j}$, then $\|\xi\|>0$ is valid for any $t$. In this way, the summation process of $\xi$ is avoided. We have proved the main theorem: Assume that $u(t, \beta)$ is single peak, then RH is valid for any $t \in[0, \infty), \beta \in(0,1 / 2]$. If using the equivalence of Lagarias (1999), the assumption of single peak can be canceled. Therefore our new thinking is that we have found the local peak-valley structure of $\xi$, which may be the geometry structure expected by Bombieri (2000), and proposed a basic framework of proving RH by geometric analysis.

\section{Keywords}

Riemann $\xi$-Function, Symmetry, Peak-Valley Structure, Single Peak, RH

\section{Introduction Symmetry}

We begin with two functions $\zeta$ and $\xi$ introduced by Riemann. Euler (1737) proved the product formula over all prime numbers

$$
\zeta(s)=\sum_{n=1}^{\infty} \frac{1}{n^{s}}=\prod_{p \in \text { prime }}\left(1-\frac{1}{p^{s}}\right)^{-1},
$$


which converges for $\operatorname{Re}(s)>1$, but diverges for $\operatorname{Re}(s) \leq 1$. Taking the complex variable $s=i t+\sigma, \sigma>1$, B. Riemann (1859) had gotten the first expression

$$
\zeta(s)=\pi^{s / 2} \Gamma^{-1}\left(\frac{s}{2}\right)\left\{\frac{1}{s(s-1)}+\int_{1}^{\infty}\left(x^{s / 2-1}+x^{-s / 2-1 / 2}\right) \psi(x) \mathrm{d} x\right\}, \psi(x)=\sum_{n=1}^{\infty} \mathrm{e}^{-n^{2} \pi x},
$$

which is analytically extended over the whole complex plane, except for $s=0,1$.

Furthermore Riemann introduced an entire function

$$
\xi(s)=\frac{1}{2} s(s-1) \pi^{-s / 2} \Gamma\left(\frac{s}{2}\right) \zeta(s), \xi(s)=\xi(1-s),
$$

and had gotten the second expression

$$
\xi(s)=\int_{1}^{\infty}\left(x^{s / 2-1}+x^{-s / 2-1 / 2}\right) f(x) \mathrm{d} x, f(x)=2 x^{2} \psi^{\prime \prime}+3 x \psi^{\prime},
$$

then proposed a proposition:

Riemann Hypothesis (RH). In the critical region

$\Omega=\{s=\sigma+i t: 0 \leq \sigma \leq 1,0 \leq t<\infty\}$, all the zeros of $\zeta(s)$ lie on the critical line $\sigma=1 / 2$, which is called the non-trivial zeros.

A lot of numerical experiments, for example [1] [2], verified that $\mathrm{RH}$ is valid. However, RH has not been proved to be valid or false in theory [3] [4] [5] [6] [7]. Why so difficult? We think there are two kinds of incorrect guide: studying $\zeta$ and using pure analysis method. We think that studying $\zeta$ is hopeless, and using pure analysis method has always met a wide gap: How to prove no zero for the infinite series? Conrey [5] pointed out that "It is my belief, $R H$ is a genuinely arithmetic question that likely will not succumb to methods of analysis". Besides, Bombieri [3] (2000) pointed out that "For them we do not have algebraic and geometric models to guide our thinking, and entirely new ideas may be needed to study these intriguing objects". Thus I felt that a unique hope is to study $\xi$ by geometric analysis.

Recall that Riemann had cleverly designed the function $\xi$ in (1.4). He took $\sigma=1 / 2$ to get the real expression

$$
\xi\left(\frac{1}{2}+i t\right)=4 \int_{1}^{\infty} \frac{\mathrm{d}\left(x^{3 / 2} \psi^{\prime}(x)\right)}{\mathrm{d} x} x^{-1 / 4} \cos \left(\frac{1}{2} t \ln x\right) \mathrm{d} x, \operatorname{Im}(\xi)=0 .
$$

This is the most important symmetry on critical line. But so far there are a few work on $\xi$, even $\xi$ is denied. Denoting $\beta=\sigma-1 / 2, \tau=\beta+i t=s-1 / 2$ and $\xi(\tau)=u+i v$, by the symmetry we have

Lemma 1 (Basic expression). For any $t \in[0, \infty)$ and $\beta \in(0,1 / 2]$, the imaginary part $v$ can be uniquely expressed by $u(t, \beta)$ in the form

$$
v(t, \beta)=-\int_{0}^{\beta} u_{t}(t, r) \mathrm{d} r .
$$

Proof. Using $v(t, \beta)=v(t, 0)+\int_{0}^{\beta} v_{\beta}(t, r) \mathrm{d} r, v(t, 0)=0$ and C-R condition $v_{\beta}=-u_{t}$, we get (1.6), which plays an important role in studying $\xi$.

Corollary 1. $|v(t, \beta)| / \beta$ is uniformly bounded with respect to $\beta \in(0,1 / 2]$.

Definition 1. For any fixed $\beta \in(0,1 / 2]$, the sub-interval $I_{j}=\left[t_{j}, t_{j+1}\right]$ is called the root-interval, if the real part $u\left(t_{j}, \beta\right)=0, u\left(t_{j+1}, \beta\right)=0$ and 
$|u(t, \beta)|>0$ inside $I_{j}$.

Definition 2. If $|u|$ in each root-interval $I_{j}$ has only one peak, called the single peak, else called the multiple peaks (Actually the multiple peak case does not exist).

In numerical experiments, we found an important fact as follows.

Proposition 1. For any fixed $\beta \in(0,1 / 2]$ and in each root-interval $I_{j}=\left[t_{j}, t_{j+1}\right]$, assume that $v(t, \beta)$ has opposite signs at $t_{j}$ and $t_{j+1}$, and $v=0$ at some inner point, then $\{|u|,|v| / \beta\}$ form local peak-valley structure, and norm $\|\xi\|=|u|+|v / \beta|>0$ in $I_{j}$, i.e. $R H$ is valid in $I_{j}$.

Using the symmetry of $\xi$ and the slope $u_{t}$ of the single peak, we have proved the assumption of the proposition 1 . Because each $t$ must lie in some $I_{j}$, thus $\|\xi\|>0$ is valid for any $t$. It proves

The main theorem. Assume that $u(t, \beta)$ is the single peak, then $R H$ is valid for any $(t, \beta) \in \Omega=[0, \infty) \times(0,1 / 2]$.

If using the equivalence of Lagarias [8], it is proved that the multiple peak case of $u(t, \beta)$ does not exist, thus a complete proof of RH can be given [9]. If only using the expression (1.4) of $\xi$, the conclusion has not be proved yet. This is an unsolved problem.

Therefore the new thinking in this paper is that we have found the local peak-valley structure of $\xi$, which may be the geometry structure expected by Bombieri (2000), and proposed a basic framework of proving RH by concise geometric analysis.

\section{Find Local Geometry Property by Computing}

The norm $|\xi|=\left(|u|^{2}+|v|^{2}\right)^{1 / 2}$ is used in complex analysis. If $\beta$ is small, then $v=O(\beta)$ is also small. To enlarge the role of $v$, in critical region $\Omega$ we introduce a strong norm

$$
\|\xi\|= \begin{cases}|u|+|v| / \beta, & \beta \in(0,1 / 2], t \in[0, \infty), \\ u(t, 0)|+| u_{t}(t, 0) \mid, & \beta \rightarrow+0, t \in[0, \infty) .\end{cases}
$$

in which $|u|$ and $|v| / \beta$ are of same order and $\|\xi\|$ is stable for $\beta>0$. Note that if $\beta=0, u\left(t_{j}, 0\right)=0, v\left(t_{j}, 0\right)=0$, then $|\xi|=0$, but $\|\xi\|>0$, because $\left|u_{t}\left(t_{j}, 0\right)\right| \neq 0$, see Figure 2 .

It is known that $\xi$ has the exponential decay [6] [9]

$$
|\xi(s)| \leq C\left(\frac{t}{2}\right)^{23 / 12+\beta / 6} \mathrm{e}^{-t \pi / 4} \ln t \text {, if }|\beta| \leq 1 / 2 .
$$

To make the figures of $\{u, v\}$, we take a changing scale

$$
M=8\left(\frac{t}{2}\right)^{23 / 12+\beta / 6} \mathrm{e}^{-t \pi / 4},
$$

and consider the curves $\{u / M, v / \beta / M\}$ and strong norm $\|\xi\| / M$, later no longer explain.

Figure 1 exhibits the curve $u(0, t)$ and 18 zeros. Figure 2 exhibits $u$ (real line) and $v / \beta$ (dot line) for $\beta=0.1$, their zeros are alternative and have 

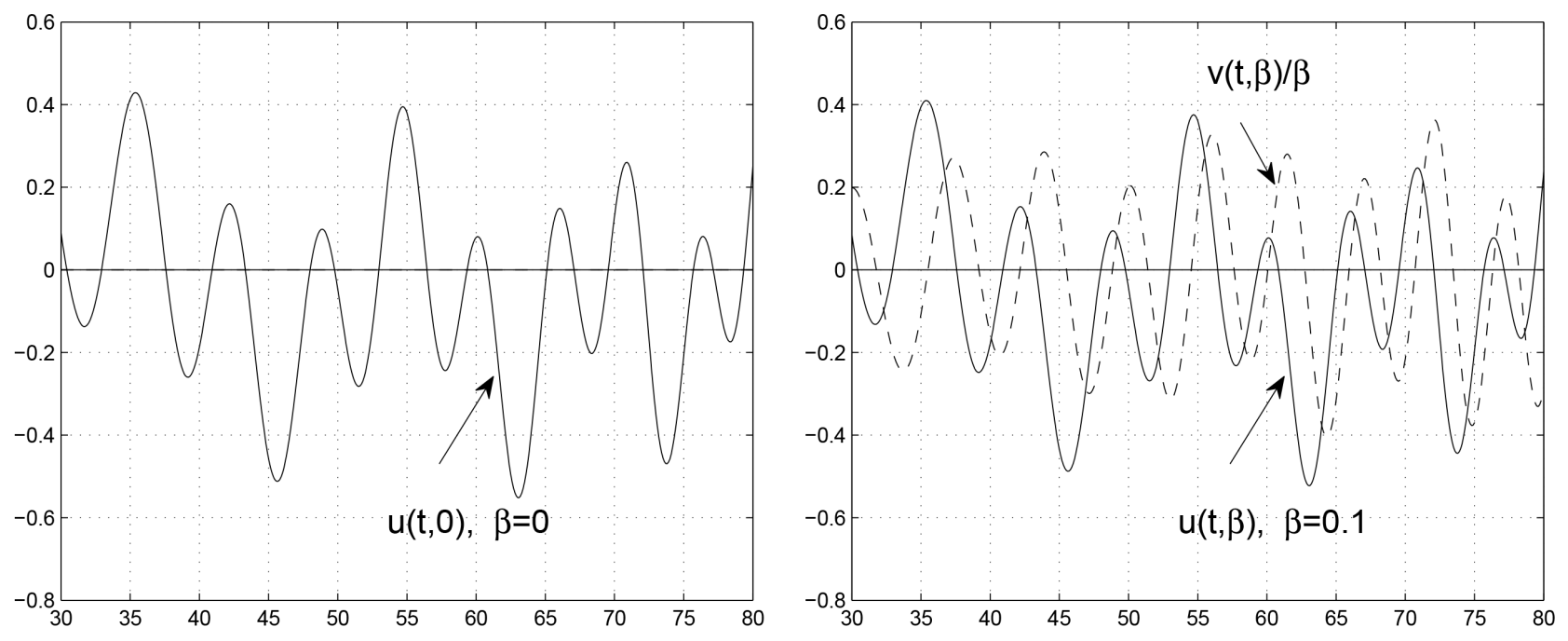

Figure 1. The curves $u(t, 0)$ and $\{u, v / \beta\}, \beta=0.1$.
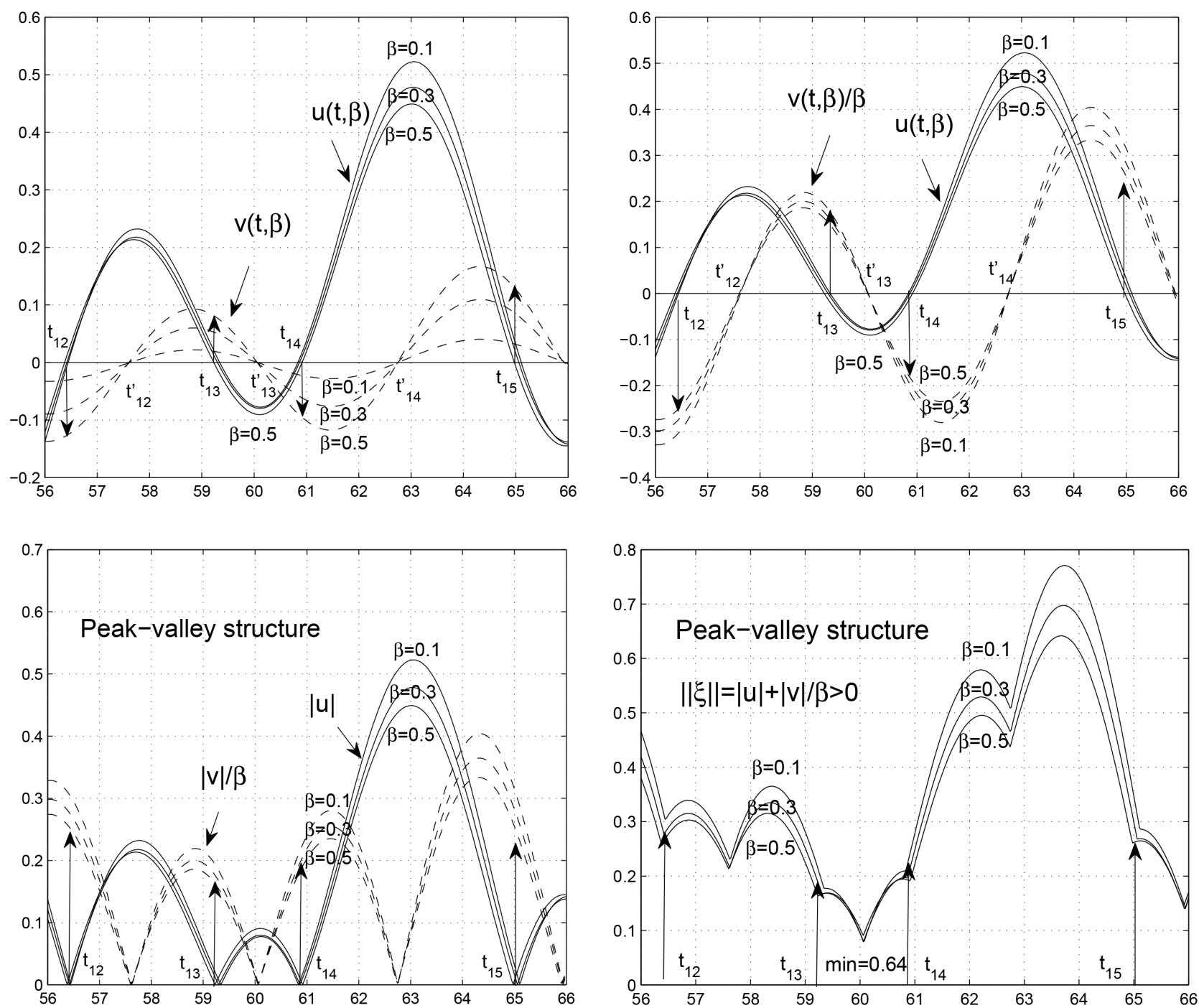

Figure 2. Figures of curves $\{u, v\},\{u, v / \beta\},\{|u|,|v| / \beta\}$ and $\|\xi\|>0$. 
"positive phase-difference", so $\|\xi\|>0$ (i.e. RH is locally valid). But we do not know how to describe it, and it's pity, is given up. At that time we always wanted to study no zero of the infinite series by the asymptotic analysis. Many times attempts have failed. After three years, we have suddenly waken up that.

"Give up method of analysis, directly study the geometry property of $\xi$ itself".

So we came back to "positive phase-difference" once again, but now we find that it is a local peak-valley structure.

We explain the local peak-valley structure in Figure 2 for $\beta=0.1,0.3,0.5$. It is seen that in each root-interval $I_{j}=\left[t_{j}, t_{j+1}\right]$, the imaginary part $v$ has opposite signs at two end-points of $I_{j}$ and $v=0$ at some inner point $t_{j}^{\prime} \in I_{j}$, then $|v| / \beta$ is a valley curve. Therefore $\{|u|,|v| / \beta\}$ form local peak-valley structure and $\|\xi\|=|u|+|v| / \beta \geq \mu\left(t_{j}, \beta\right)>0$. Although each lower bound $\mu\left(t_{j}, \beta\right)$ is different for different $I_{j}$ and $\beta>0$, but which is always positive.

\section{Local Peak-Valley Structure in Single Peak Case}

For fixed $\beta \in(0,0.5]$ the zeros $t_{j}$ of $u(t, \beta)$ form an infinite sequence dependent on $\beta$

$$
0<t_{1}<\cdots<t_{j-1}<t_{j}<t_{j+1}<t_{j+2}<\cdots \rightarrow \infty
$$

We shall take them as the base and consider only single peak case.

The slope of single peak. For any $\beta \geq 0$, there are $u_{t}>0$ from negative peak to positive one, and $u_{t}<0$ from positive peak to negative one.

Theorem 1 (single peak case). Assume that $u(t, \beta)$ is the single peak for any $\beta \in(0,1 / 2]$, then $\|\xi\|>0$ for any $(t, \beta) \in R=\{[0, \infty) \times(0,1 / 2]\}$.

Proof. Below it is enough to discuss $u>0$ inside each root-interval $I_{j}=\left[t_{j}, t_{j+1}\right]$. For any fixed $\beta>0$, using Lemma 1 , we consider two cases as follows.

As $u_{t}>0$ near the left node $t_{j}$, we have

$$
\left\{\begin{array}{l}
v\left(t_{j}, \beta\right) / \beta=-\frac{1}{\beta} \int_{0}^{\beta} u_{t}\left(t_{j}, r\right) \mathrm{d} r<0, \\
\lim _{\beta \rightarrow+0} v\left(t_{j}, \beta\right) / \beta=-u_{t}\left(t_{j}, 0\right)<0 .
\end{array}\right.
$$

As $u_{t}<0$ near the right node $t_{j+1}$, similarly

$$
\left\{\begin{array}{l}
v\left(t_{j+1}, \beta\right) / \beta=-\frac{1}{\beta} \int_{0}^{\beta} u_{t}\left(t_{j+1}, r\right) \mathrm{d} r>0, \\
\lim _{\beta \rightarrow+0} v\left(t_{j+1}, \beta\right) / \beta=-u_{t}\left(t_{j+1}, 0\right)>0 .
\end{array}\right.
$$

which are valid and numerically stable for $\beta \in(0,1 / 2]$.

Because $v(t, \beta)$ has opposite signs at two end-points in $I_{j}$, there certainly exists an inner point $t_{j}^{\prime}=t_{j}^{\prime}(\beta)$ such that $v\left(t_{j}^{\prime}, \beta\right)=0$. Thus $\{|u|,|v| / \beta\}$ form a local peak-valley structure. We regard $\|\xi(t, \beta)\|$ as a continuous function with respect to $(t, \beta)$, which certainly has a positive lower bound independent of $t \in I_{j}$, 


$$
\min _{t \in I_{j}}\|\xi(t, \beta)\|=\mu\left(t_{j}, \beta\right)>0, \beta \in(0,0.5] .
$$

This is a fine local geometric analysis.

Thus in each root-interval $I_{j}=\left[t_{j}, t_{j+1}\right]$, we can determine a positive lower bound $\mu\left(t_{j}, \beta\right)>0$, which form the positive infinite sequence

$$
\mu\left(t_{1}, \beta\right), \mu\left(t_{2}, \beta\right), \cdots, \mu\left(t_{j}, \beta\right), \mu\left(t_{j+1}, \beta\right), \cdots
$$

Because each $t$ must lie in some $I_{j}$, thus $\|\xi\|>0$ for any $t$. In this way, we have completely avoided the summation process of the infinite series $\xi$. Theorem 1 is proved.

In the theorem 1, we are anxious that when $\beta$ increases, if some root-interval will be contracted to a point so that $u=v=0$ ? This worry is denied by the following theorem 2.

Theorem 2. Assume that for $\beta_{0} \in[0,1 / 2]$, the root-interval $I_{j}^{0}=\left[t_{j}^{0}, t_{j+1}^{0}\right]$ is far less than two adjacent root-intervals, then when $\beta$ increases, the corresponding root-interval $I_{j}=\left[t_{j}, t_{j+1}\right]$ will enlarge, rather than decrease.

Proof. Assume that for $\beta_{0} \geq 0, u\left(t, \beta_{0}\right)$ has a solitary small root-interval $I_{j}^{0}=\left[t_{j}^{0}, t_{j+1}^{0}\right], u\left(t_{j}^{0}, \beta_{0}\right)=0, u\left(t_{j+1}^{0}, \beta_{0}\right)=0$, and attains maximum value $u\left(t^{\prime}, \beta_{0}\right)=\varepsilon>0$ at some inner point $t^{\prime} \in I_{j}^{0}$, and $u_{t}=0, u_{t t}<0$ at $t^{\prime}$. We consider a little large sub-interval $I \supset I_{j}^{0}$, in which $u_{t t}<0$, and $u\left(t, \beta_{0}\right) \leq \varepsilon$ is a convex curve upward. So $u_{t}>0$ for $t<t^{\prime}$, and $u_{t}<0$ for $t>t^{\prime}$.

Consider a small increment $\beta-\beta_{0}=\alpha>0$. By basic expression in $I$ we have

$$
v_{t}(t, \beta)=-\int_{0}^{\beta} u_{t t}(t, r) \mathrm{d} r>0, \text { as } u_{t t}(t, r)<0,
$$

and

$$
\begin{aligned}
u(t, \beta)-u\left(t, \beta_{0}\right) & =\int_{\beta_{0}}^{\beta} u_{\beta}(t, r) \mathrm{d} r=\int_{\beta_{0}}^{\beta} v_{t}(t, r) \mathrm{d} r, \text { as } u_{\beta}=v_{t}, \\
& =-\int_{\beta_{0}}^{\beta}\left(\int_{0}^{r} u_{t t}\left(t, r^{\prime}\right) \mathrm{d} r^{\prime}\right) \mathrm{d} r=d>0 .
\end{aligned}
$$

Thus the real part $u(t, \beta)$ has removed $u\left(t, \beta_{0}\right)$ in parallel by a distance $d>0$ toward its convex direction. Due to $u\left(t, \beta_{0}\right)<0$ outside $I_{j}^{0}$, there are certainly a left node $t_{j}=t_{j}(\beta)<t_{j}^{0}$ with $v\left(t_{j}, \beta\right)=0$ and a right node $t_{j+1}=t_{j+1}(\beta)>t_{j+1}^{0}$ with $u\left(t_{j+1}, \beta\right)=0$. Then $u(t, \beta)$ is a positive peak curve inside the enlarged interval $I_{j}=\left[t_{j}, t_{j+1}\right] \supset I_{j}^{0}$.

Besides, by basic expression we know that

$$
v\left(t_{j}, \beta\right)<0\left(\text { as } u_{t}>0\right), v\left(t_{j+1}, \beta\right)>0\left(\text { as } u_{t}<0\right),
$$

i.e., $|v| / \beta$ still is a valley curve and $\|\xi\| \geq \mu\left(t_{j}, \beta\right)>0$ in $I_{j}$. Theorem 2 is proved.

From Theorem 2, we found a wonderful property:

When $\beta \geq 0$ increases, these root-intervals have a tendency to get more unform.

This property makes RH be still valid when $\beta$ increases (e.g. RH is valid for $\beta=1 / 2$ ). 
Finally summarizing the theorem 1 and theorem 2 our main theorem is proved.

Remark 1. We have a question: Is the $u(t, 0)$ single peak? Through large scale computation, Lune et al. [1] [2] have pointed out that all zeros of $u$ on critical line $0 \leq t \leq 5.6 e+8$ are single, no double. We can see in several curve figures that the $u(t, 0)$ is single peak, no multiple peak. This is heuristic, but not proof.

Remark 2. It is proved [9] that multiple peak case does not exist. For this, we have used Lagarias's positivity [8] $\operatorname{Re}\left(\frac{\xi^{\prime}}{\xi}\right)>0$ to judge that $\psi=u v_{t}-v u_{t}>0$ is not satisfied at multiple peak point. While Lagarias have used Hadamard's formula independent of (1.4) to prove the equivalence. Can we directly get the judgment by (1.4)? No success, because we can get only linear relations by (1.4), but $\psi$ is a quadratic form. One unsolved question remains.

\section{Acknowledgements}

The author expresses sincere thanks to the reviewer for his valuable comments, suggestion and kind encouragement.

\section{Conflicts of Interest}

The author declares no conflicts of interest regarding the publication of this paper.

\section{References}

[1] Lune, J. and Riele, H. (1983) On the Zeros of the Riemann Zeta Function in the Critical Strip. Part 3. Mathematics of Computation, 41, 759-767. https://doi.org/10.1090/S0025-5718-1983-0717719-3

[2] Lune, J., Riele, H. and Winter, D. (1986) On the Zeros of the Riemann Zeta Function in the Critical Strip. Part 4. Mathematics of Computation, 46, 667-681. https://doi.org/10.1090/S0025-5718-1986-0829637-3

[3] Bombieri, E. (2000) Problems of the Millennium: The Riemann Hypothesis.

[4] Borwein, P., Choi, S., Rooney, B. and Weirathmuller, A. (2006) The Riemann Hypothesis. Springer, New York.

[5] Conrey, J. (2003) The Riemann Hypothesis. Notices of the AMS, 50, 341-353.

[6] Edwards, H. (2001) Riemann's Zeta Function. Dover Publication, Inc., Mineola.

[7] Smale, S. (1998) Mathematical Problems for Next Century. The Mathematical Intelligencer, 20, 7-15. https://doi.org/10.1007/BF03025291

[8] Lagarias, J. (1999) On a Positivity Property of the Riemann $\zeta$-Function. Acta Arithmetica, 89, 213-234. https://doi.org/10.4064/aa-89-3-217-234

[9] Chen, C.M. (2020) Local Geometric Proof of Riemann Hypothesis. 